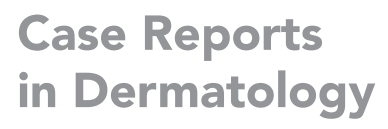

\title{
Distant Resolution of Actinic Keratosis following Cryosurgery: An Unusual Phenomenon
}

\author{
Karim Saleh \\ Division of Dermatology and Venereology, Department of Clinical Sciences, Skåne \\ University Hospital, Lund University, Lund, Sweden
}

\section{Keywords}

Cryosurgery · Cryotherapy · Actinic keratosis $\cdot$ Immune response

\begin{abstract}
Early after the introduction of cryosurgery to clinical practice, there were reports of metastasis regressing after cryosurgery of a primary tumour, mainly prostate and breast cancer, suggesting a systemic immunological effect to a local reaction. Colleagues within dermatology have occasionally experienced similar systemic effects following cryosurgery. However, published reports of such cases are lacking. In this case, we report a photographed distant resolution of an actinic keratosis (AK) on 68-year-old woman's arm following cryosurgery of another AK on the same arm.
\end{abstract}

\section{Introduction}

Immune response activation following cryosurgery is controversial and not fully explored. In this case, we report our experience of a distant actinic keratosis (AK) resolution following cryosurgery of another AK.

\section{Case Presentation}

A 68-year-old woman with a history of multiple AKs, squamous cell carcinomas, and basal cell carcinomas has been a patient at our department for the last 5 years. She has no other medical conditions or psychiatric history and no medications. 
The patient presented to our department recently with 2 clinically diagnosed AKs on her right lower arm. According to the patient and our medical records, only the distal thicker AK was treated. Treatment consisted of routine cryosurgery spraying liquid nitrogen on the lesion using 2 thawing cycles lasting $30 \mathrm{~s}$ each. The patient described an initial reaction on the distal AK a couple of hours after the treatment. Approximately $24 \mathrm{~h}$ later, she developed a reaction distant to the treated lesion. This reaction was around the second AK located approximately 10 $\mathrm{cm}$ proximal to the treated lesion. The patient captured a photo taken approximately $36 \mathrm{~h}$ after cryosurgery (Fig. 1) and 10 days post-cryosurgery (Fig. 2). Both lesions had similar reactions and healing times and ultimately both AKs cleared. The patient also sent us a photo months post-cryosurgery (Fig. 3). She mentioned her son had a similar phenomenon, in which a surgical removal of a suspected nevus resulted in the disappearance of numerous nevi.

\section{Discussion}

Cryosurgery which involves the use of extreme cold temperatures on tissue started in England between 1845 and 1851 when iced saline solutions were used [1]. It was initially developed as a method of anaesthesia, but it was soon noted it had an effect on the viability

Fig. 1. Distant cryosurgery reaction in an untreated AK proximal to the treated lesion. Photograph taken by the patient $36 \mathrm{~h}$ after cryosurgery. AK, actinic keratosis.

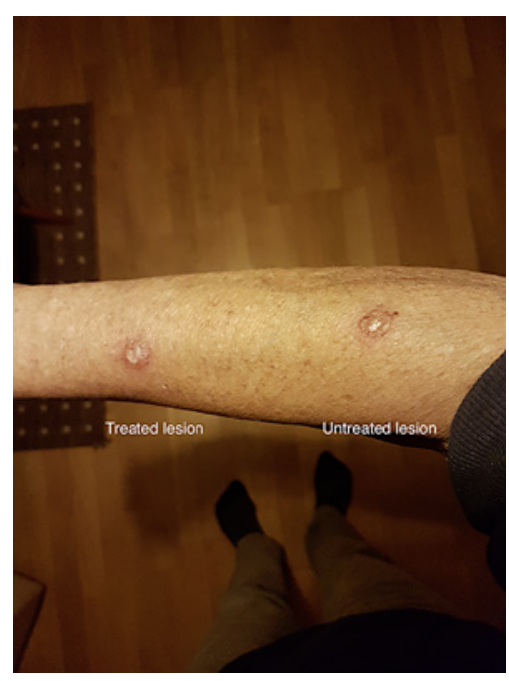

Fig. 2. Distant cryosurgery reaction in an untreated AK proximal to the treated lesion. Photograph taken by the patient 10 days after cryosurgery. AK, actinic keratosis.

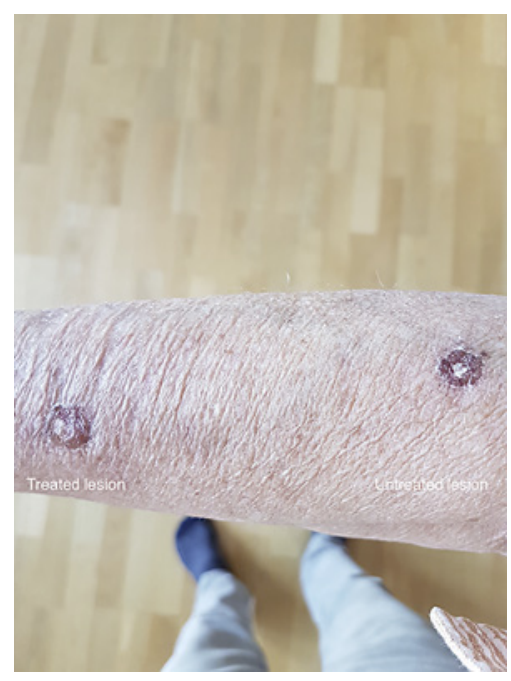


Fig. 3. Fully healed lesions photographed months after cryosurgery.

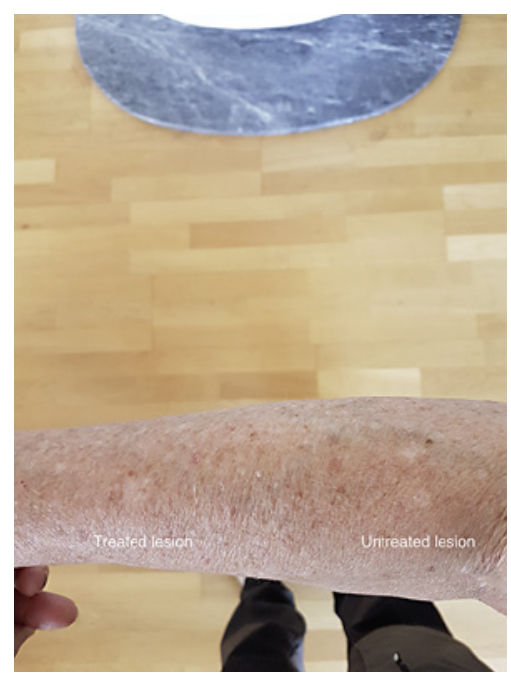

of cancer cells and thus the idea of destroying diseased tissue through cryosurgery was born. Initially, it was practised on superficial tumours due to a limited temperature between -18 and $-24^{\circ} \mathrm{C}$, but with engineering developments allowing liquification of oxygen air and nitrogen, temperatures around $-190^{\circ} \mathrm{C}$ were attainable which increased clinical applicability. Over the past few decades, cryosurgery has been used to treat malignancies of the skin, prostate, liver, breast, lung, and bone, and more applications are being studied [2]. Cryosurgery advantages include the minimally invasive nature of the treatment, less damage to surrounding structures, patient comfort, speed, the cost of therapy, and improved cosmetic results.

Cryosurgery is known to induce tissue necrosis through several mechanisms including apoptosis, cellular injury, vessel thrombosis, and immune response activation [3]. The latter is still controversial and not clearly studied. Early after the introduction of cryosurgery to clinical practice, there were reports of metastasis regressing after cryosurgery of a primary tumour, mainly prostate and breast cancer, suggesting a systemic immunological reaction to a local reaction $[3,4]$.

It is hypothesized that cryosurgery causes release of DNA, RNA, uric acid, and other intracellular elements from the destructed cells. These elements activate tolllike receptors, triggering subsequent immunologic cascades. An innate immune response is generated initially, resulting in early infiltration of granulocytes, monocytes, and macrophages. An adaptive immune response follows, in which dendritic cells travel to regional lymph nodes to undergo maturation and antigen presentation [5]. Inducing the immune system by cryosurgery has sometimes been termed cryoimmunology and is gaining current research [6]. In a few reports, it has been tested on urological and bone tumours in which a locally treated tumour yielded a systemic effect on metastasis $[7,8]$.

Colleagues within dermatology have occasionally experienced similar systemic effects following cryosurgery but to the best of the author's knowledge, none of these clinical reports have been published. We hypothesize our patient experienced a systemic immunological reaction in which a distant AK cleared but apparently further studies are required to evaluate this hypothesis and explore whether systemic effect of local cryosurgery could be attained.

\section{Karger'}




\section{Statement of Ethics}

The written informed consent was obtained from the patient for publication of this case report and any accompanying images. A copy of this consent is with the corresponding author. The study was conducted according to the Declaration of Helsinki.

\section{Conflict of Interest Statement}

The author has no conflicts of interest to declare.

\section{Funding Sources}

The author did not receive any funding.

\section{Author Contributions}

K.S. was the only contributor to this case report.

\section{References}

1 Bird HM, Arnott J. A pioneer in refrigeration analgesia. Anaesthesia. 1949;4:10-7.

2 Sabel MS. Cryo-immunology: a review of the literature and proposed mechanisms for stimulatory versus suppressive immune responses. Cryobiology. 2009 Feb;58(1):1-11.

3 Soanes WA, Ablin RJ, Gonder MJ. Remission of metastatic lesions following cryosurgery in prostatic cancer: immunologic considerations. J Urol. 1970 Jul;104(1):154-9.

4 Gursel E, Roberts M, Veenema RJ. Regression of prostatic cancer following sequential cryotherapy to the prostate. J Urol. 1972 Dec;108(6):928-32.

5 Kasuya A, Ohta I, Tokura Y. Structural and immunological effects of skin cryoablation in a mouse model. PLoS One. 2015;10(3):e0123906.

6 Korpan NN, Goltsev AN, Dronov OI, Bondarovych MO. Cryoimmunology: opportunities and challenges in biomedical science and practice. Cryobiology. 2021 Feb 24.

7 Sidana A, Chowdhury WH, Fuchs EJ, Rodriguez R. Cryoimmunotherapy in urologic oncology. Urology. 2010 May; 75(5):1009-14.

8 Nishida H, Yamamoto N, Tanzawa Y, Tsuchiya H. Cryoimmunology for malignant bone and soft-tissue tumors. Int J Clin Oncol. 2011 Apr;16(2):109-17.

\section{Karger'}

